\title{
Cannabinoid-Verordnungen in der Praxis
}

\author{
Autorin: Isabel Kuhlen
}

\section{ZUSAMMENFASSUNG}

Seit März 2017 kann der Wirkstoff Cannabis unter gesetzlich festgelegten Voraussetzungen verordnet werden. Allerdings ist die Verordnung genehmigungspflichtig durch die GKV. In der Praxis bestehen häufig noch Unsicherheiten, nicht alle Anträge werden genehmigt oder die Genehmigung zeitlich befristet, was nach einem aktuellen Eilverfahren nicht mehr zulässig ist.

Der Artikel erläutert die gesetzlichen Grundlagen, die Voraussetzungen für die Verordnungsfähigkeit von Cannabinoiden, was bei der Antragstellung zu beachten ist und welche Fristen vonseiten der GKV für die Genehmigung gelten.

\section{Schlüsselwörter}

Cannabinoide, Cannabis, Cannabis-Gesetz, Antragstellung, Verordnungsfähigkeit

\section{ABSTRACT}

Since March 2017, the active ingredient cannabis can be prescribed under conditions stipulated by law. However, the prescription is subject to approval by the statutory health insurances. In practice however, there are still many uncertainties, not all applications are approved, or the approval has a time limitation, which is no longer allowed according to current expedited proceedings.

The article explains the legal basics, the conditions for the prescribability of cannabinoids, what has to be taken into account when making an application, and which deadlines apply for the approval on the part of the statutory health insurances.

\section{Keywords}

Cannabinoids, cannabis, cannabis law, application, prescribability

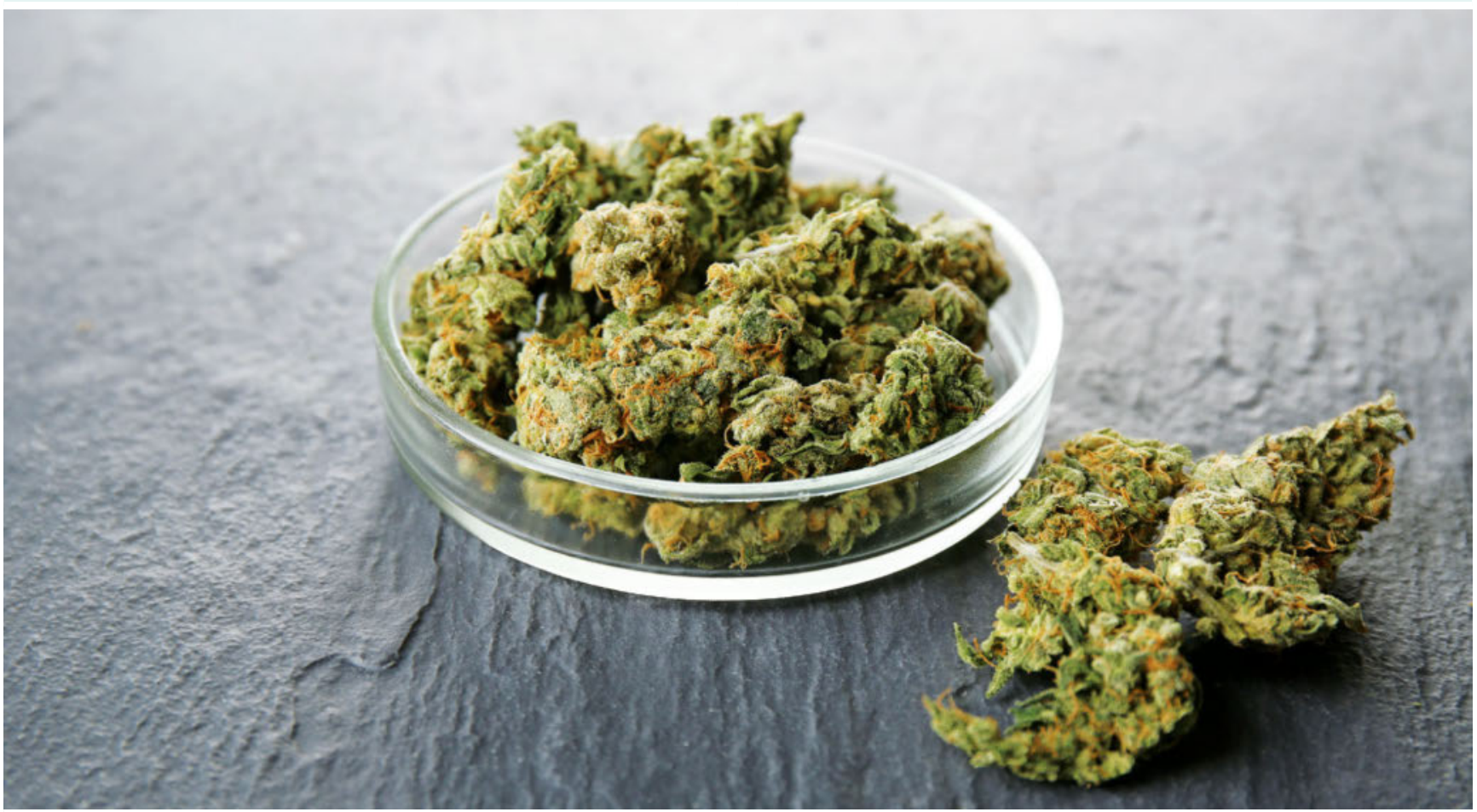

•Abb. 1 Die Verordnung von Cannabinoiden ist seit März 2017 gesetzlich geregelt. @ Africa Studio/Adobe Stock

Durch das im März 2017 in Kraft getretene sog. „CannabisGesetz“ ist der Wirkstoff Cannabis unter gesetzlich festgelegten Voraussetzungen betäubungsmittelrechtlich verkehrs- und verordnungsfähig geworden. Zudem wurde die Verordnungsähigkeit mehrerer Cannabinoide zu Lasten der GKV gesetzlich normiert. Obwohl inzwischen mehr als ein Jahr vergangen ist, zeigt sich in der täglichen Praxis, dass noch verschiedene Punkte klärungsbedürftig sind.

\section{Gesetzliche Grundlage}

Das „Gesetz zur Änderung betäubungsmittelrechtlicher und anderer Vorschriften“ ist am 10.3.2017 in Kraft getreten. Aufgrund der Inhalte dieses neuen Gesetzes ist nunmehr einerseits Cannabis (Marihuana, Pflanzen und Pflanzenteile der zur Gattung Cannabis gehörenden Pflanze) unter der Voraussetzung, dass es aus einem Anbau, der zu medizinischen Zwecken unter staatlicher Kontrolle er- 
folgt bzw. in Zubereitungen, die als Fertigarzneimittel zugelassen sind - betäubungsmittelrechtlich verkehrs- und verordnungsfähig.

Weiterhin hat der Gesetzgeber besondere Voraussetzungen festgelegt, bei deren Einhaltung Cannabinoide grundsätzlich zu Lasten der Gesetzlichen Krankenversicherung (GKV) verordnungsfähig sind. Der neue §31 Abs.6 SGB V hat folgenden Wortlaut:

„Versicherte mit einer schwerwiegenden Erkrankung haben Anspruch auf Versorgung mit Cannabis in Form von getrockneten Blüten oder Extrakten in standardisierter Qualität und auf Versorgung mit Arzneimitteln mit den Wirkstoffen Dronabinol oder Nabilon, wenn 1. eine allgemein anerkannte, dem medizinischen Standard entsprechende Leistung

a) nicht zur Verfügung steht oder

b) im Einzelfall nach der begründeten Einschätzung der behandelnden Vertragsärztin oder des behandelnden Vertragsarztes unter Abwägung der zu erwartenden Nebenwirkungen und unter Berücksichtigung des Krankheitszustandes der oder des Versicherten nicht zur Anwendung kommen kann,

2. eine nicht ganz entfernt liegende Aussicht auf eine spürbare positive Einwirkung auf den Krankheitsverlauf oder auf schwerwiegende Symptome besteht.

Die Leistung bedarf bei der ersten Verordnung für eine Versicherte oder einen Versicherten der nur in begründeten Ausnahmefällen abzulehnenden Genehmigung der Krankenkasse, die vor Beginn der Leistung zu erteilen ist. Verordnet die Vertragsärztin oder der Vertragsarzt die Leistung nach Satz 1 im Rahmen der Versorgung nach $\S 37 b$, ist über den Antrag auf Genehmigung nach Satz 2 abweichend von § 13 Absatz 3a Satz 1 innerhalb von drei Tagen nach Antragseingang zu entscheiden. “

Es existieren somit nunmehr gesetzliche Rahmenvorgaben, die eine Verordnung von Cannabinoiden zu Lasten der GKV in besonderen Fällen ermöglichen.

\section{In der Praxis unterschiedlich bewertete Vorgaben}

Der Gesetzgeber hat zwar im Wortlaut des Gesetzes bereits besondere Voraussetzungen für eine Verordnungsfähigkeit von Cannabinoiden zu Lasten der GKV festgelegt. Dennoch werden bestimmte Einzelfragen von den beteiligten Parteien unterschiedlich bewertet. Die Krankenkassen auf der einen Seite und die Ärzte und Versicherten auf der anderen Seite vertreten zum Teil unterschiedliche Auffassungen. Betroffen hiervon sind bereits Einzelheiten der gesetzlich fixierten Vorgaben.

\section{Voraussetzung: Vorliegen einer „schwerwiegen- den Erkrankung“}

Der Gesetzgeber hat die Verordnung eines Cannabinoids zu Lasten der GKV an die Voraussetzung gebunden, dass eine „schwerwiegende Erkrankung“ vorliegt. Das Vorliegen einer solchen Erkrankung im Sinne der gesetzlichen Regelung muss im Einzelfall geprüft werden. Grundsätzlich ist insoweit, das hat das Landessozialgericht Hessen [1] bereits ausdrücklich bestätigt, die Definition des $\S 33$ der Arzneimittel-Richtlinien (AM-RL) zur Auslegung dieser Vorgabe anzuwenden. §33 AM-RL hat folgenden Wortlaut:

„Eine Krankheit ist schwerwiegend, wenn sie lebensbedrohlich ist oder aufgrund der Schwere der durch sie verursachten Gesundheitsstörung die Lebensqualität auf Dauer nachteilig beeinträchtigt ist. “

Wichtig ist in der Praxis daher bei der Antragstellung, dass eine etwaig vorliegende dauerhafte Beeinträchtigung der Lebensqualität im Detail dargelegt wird. Nur wenn der Krankenkasse bei der Antragstellung dargelegt wird, dass eine dauerhafte Beeinträchtigung der Lebensqualität vorliegt, kann diese Tatsache bei der Prüfung der Genehmigungsfähigkeit durch die Krankenkasse auch Berücksichtigung finden.

In der Rechtsprechung des Bundessozialgerichts [2] zum sog. „Off-label-use“ sind folgende Krankheitsbilder bereits als schwerwiegende Erkrankungen anerkannt worden:

- Multiple Sklerose

- Tumorleiden und

- AIDS.

Darüber hinaus hat das Bundessozialgericht auch „eine Myopathie wegen Myoadenylate-Deaminase-Mangels“ [3] und den „Zustand nach Subarachnoidalblutung und des daraus resultierenden Hirntraumas “ [4] als schwerwiegende Erkrankung anerkannt.

Zudem werden z. B. auch folgende Erkrankungen von der Krankenkasse in der Regel als schwerwiegend anerkannt:

- Endgradig chron. schwerste Schmerzen (VAS 8-10) mit Aufhebung der Schlafarchitektur und einer depressiven Entwicklung mit latenter Suizidalität.

- Kachexie-Syndrom bei fortgeschrittenem Tumorleiden in Kombination mit schwer kontrollierbaren viszeralen Schmerzen.

\section{Voraussetzung: Fehlende Möglichkeit der Thera- pie durch allgemein anerkannte, dem medizinischen Standard entsprechende Alternativtherapien}

Eine weitere gesetzlich festgelegte Voraussetzung der Verordnungsfähigkeit von Cannabinoiden zu Lasten der GKV sorgt in der Praxis für die meisten Streitfälle:

Häufig werden Anträge mit der Begründung der Krankenkassen zunächst abgelehnt, dass die Ungeeignetheit potenzieller Alternativtherapien nicht ausreichend dargelegt 
worden wäre. Durch den Gesetzgeber wurde insoweit bereits ausdrücklich festgelegt, dass der Patient nicht alle theoretisch denkbaren Alternativtherapien ausprobiert haben muss, damit die Verordnung eines Cannabinoids durch seinen Arzt erfolgen kann. Ausreichend ist es nach dem Wortlaut des Gesetzes, wenn nach der subjektiven Einschätzung des behandelnden Arztes eine Alternativtherapie nicht erfolgversprechend ist. In diesem Fall braucht eine potenziell mögliche Therapie nicht bei dem Patienten angewendet worden zu sein, um eine Verordnungsfähigkeit des Cannabinoids veranlassen zu können.

Diese Vorgabe wurde auch bereits gerichtlich bestätigt. Das Sozialgericht Bremen [5] hat in einem Eilverfahren bestätigt, dass die Erprobung sämtlicher alternativer Behandlungsmöglichkeiten nicht von den Krankenkassen gefordert werden kann. Sinngemäß hielt das Gericht fest, dass ein Versicherter nicht verpflichtet ist, sämtliche alternativen Behandlungsmöglichkeiten an sich ausprobieren zu lassen und langjährig schwerwiegende Nebenwirkungen zu ertragen, damit eine Therapie unter Verwendung eines Cannabinoids von seiner Krankenkasse genehmigt werden kann.

Die Krankenkassen [6] gehen bisher davon aus, dass eine potenzielle Alternativtherapie nicht anwendbar ist, wenn folgende Voraussetzungen nachweisbar sind:

- Die Therapie wurde bereits erfolglos durchgeführt.

- Es bestehen Kontraindikationen.

- Es sind medizinisch begründet und nachvollziehbar nicht tolerierbare Nebenwirkungen zu erwarten.

Hinsichtlich des Vorliegens dieser Vorgaben bedarf es einer medizinisch begründeten und nachvollziehbaren Einschätzung des behandelnden Vertragsarztes. Zur Begründung einer solchen Einschätzung wird von den Krankenkassen z. B. gefordert, dass Inhalte der Fachinformation die Einschätzung unterstreichen sollen. Es wird z. B. von den Krankenkassen vertreten, dass eine Alternativtherapie nicht zur Anwendung kommen könne, wenn bereits eine Nebenwirkung aufgetreten ist, die in der Fachinformation angegeben wird und welche auch die Anwendung anderer Wirkstoffe ausschließt.

Ebenfalls anerkannt werden sollen Fällen, in denen der Versicherte unter einer Erkrankung leidet, zu der es „besondere Warnhinweise und Vorsichtsmaßnahmen “ für die Anwendung in der Fachinformation gibt, sodass mit einer Gefährdung bei der Therapie zu rechnen sei.

Besondere Sorgfalt ist bei der Geltendmachung von Nebenwirkungen erforderlich. Weil die Krankenkassen [6] bereits darauf hinweisen, dass nach ihrer Einschätzung eine Nebenwirkung nicht plausibel sei, die in der Fachinformation nicht genannt werde und für die der geltend machende Arzt keine Meldung nach der Berufsordnung für Ärzte vornimmt, müssen die betroffenen Ärzte hier aufpassen.
Aufgrund der Regelung in $\S 6$ der (Muster)-Berufsordnung sind Ärzte verpflichtet, die ihnen aus ihrer ärztlichen Behandlungstätigkeit bekanntwerdenden unerwünschten Wirkungen von Arzneimitteln der Arzneimittelkommission der deutschen Ärzteschaft und bei Medizinprodukten auftretende Vorkommnisse der zuständigen Behörde mitzuteilen [7]. Die Krankenkassen könnten daher bei nicht durchgeführter Meldung einer Nebenwirkung bei der Anerkennung von nicht tolerablen Nebenwirkungen Probleme verursachen. Dies einerseits indem die Anerkennung als Grund für die Nichtanwendbarkeit einer Alternativtherapie verweigert wird. Andererseits ist nicht auszuschließen, dass auch versucht wird, berufsrechtliche Konsequenzen zu veranlassen, wenn die Beobachtung einer solchen Nebenwirkung geltend gemacht wurde, aber keine entsprechende Meldung erfolgt ist. Es empfiehlt sich daher eine detaillierte Dokumentation auftretender Nebenwirkungen zu Nachweiszwecken.

\section{Voraussetzung: Bestehen einer nicht ganz entfernt liegenden Aussicht auf eine spürbare positive Einwir- kung auf den Krankheitsverlauf oder auf schwerwie- gende Symptome}

Auch diese Verordnungsvoraussetzung hat der Gesetzgeber recht detailliert geregelt. Es kann nach dem ausdrücklichen Wortlaut des Gesetzes entweder die Behandlung der Grunderkrankung oder auch „nur“ eines schwerwiegenden Symptoms angestrebt werden. Daher sollte im Rahmen der Antragstellung zunächst klargestellt werden, auf welches Behandlungsziel die Behandlung abzielt.

Unterschiedliche Rechtsauffassungen gibt es hier bei der Frage, unter welchen Voraussetzungen ein Symptom „schwerwiegend“ ist. Um den Anwendungsbereich einzuschränken, versuchen die Krankenkassen [6] bisher eine enge Auslegung dieses Begriffes durchzusetzen: Sie machen eine Auslegung in Anlehnung an die Definition der „schwerwiegenden Nebenwirkungen“ bei Arzneimitteln geltend. Der Begriff „schwerwiegende Nebenwirkung“ ist ein unbestimmter Rechtsbegriff, der durch das Arzneimittelgesetz [9] definiert wird. Nach dem Wortlaut des Gesetzes handelt es sich bei einer schwerwiegenden $\mathrm{Ne}$ benwirkung um Nebenwirkungen, „die tödlich oder lebensbedrohlich sind, eine stationäre Behandlung oder Verlängerung einer stationären Behandlung erforderlich machen, zu bleibender oder schwerwiegender Behinderung, Invalidität, kongenitalen Anomalien oder Geburtsfehlern führen.“

Wäre eine so enge Auslegung, die erfordert, dass durch das Symptom zumindest ein Krankenhausaufenthalt notwendig wurde oder verlängert werden musste, korrekt, würden Cannabinoide nur in wesentlich selteneren Fällen zu Lasten der GKV eingesetzt werden können als dies vom Gesetzgeber vorgesehen war. Daher ist die Definition des Begriffs „schwerwiegendes Symptom“ in Anlehnung an die 
Definition der schwerwiegenden Erkrankung in den Arzneimittelrichtlinien wesentlich sachdienlicher. Diese definieren [9] eine Erkrankung als „schwerwiegend“, „wenn sie lebensbedrohlich ist oder aufgrund der Schwere der hierdurch verursachten Gesundheitsstörung die Lebensqualität auf Dauer nachhaltig beeinträchtigt wird.“

Diese Definition entsprechend angewendet, wäre es sinnvoll, dass die Rechtsprechung in §31 Abs.6 SGB V ergänzend ein Symptom als „schwerwiegend“ definiert, „wenn es lebensbedrohlich ist oder aufgrund der Schwere der hierdurch verursachten Gesundheitsstörung die Lebensqualität auf Dauer nachhaltig beeinträchtigt wird“. Dann läge ein schwerwiegendes Symptom bereits vor, wenn die Lebensqualität auf Dauer nachteilig beeinträchtigt wird, ohne dass insoweit ein Krankenhausaufenthalt daraus resultieren müsste.

Solange es zur Auslegung dieser Voraussetzung keine gefestigte Rechtsprechung gibt, sollten Ärzte in jedem Fall darauf achten, dass bei der Antragstellung die zu behandelnden Symptome in ihrer gesamten Tragweite und insbesondere im Hinblick auf die Auswirkungen auf die Lebensqualität des Patienten geschildert werden. Soweit ein Krankenhausaufenthalt im Raum stehen sollte, ist es sinnvoll, ausdrücklich darauf hinzuweisen.

Zum Nachweis für eine nicht ganz entfernt liegende Aussicht auf eine spürbare Einwirkung auf den Krankheitsverlauf oder schwerwiegende Symptome ist nach der bisherigen Rechtsprechung kein Wirksamkeitsnachweis nach den Maßstäben der evidenzbasierten Medizin erforderlich. Vielmehr kommen als Beurteilungsgrundlage nach der Rechtsprechung des LSG Hessen [10] - wenn höherwertige Studien fehlen - auch folgende Nachweise in Betracht:

- Assoziationsbeobachtungen,

- pathophysiologische Überlegungen,

- deskriptive Darstellungen,

- Einzelfallberichte,

- Meinungen anerkannter Experten,

- Berichte von Expertenkomitees und

- Konsensuskonferenzen.

Vorsicht ist geboten, wenn eine Behandlung in der Fachdiskussion bereits ablehnend bewertet wurde. Da die Rechtsprechung des BSG [11] in diesem Fall bereits festgestellt hat, dass insoweit kein ausreichender Wirksamkeitsnachweis vorliegt, ist auch bei Antragstellungen nach §31 Abs.6 SGB V nicht zu empfehlen, auf Einzelmeinungen eines Arztes zu verweisen, wenn diese Meinung in der Fachdiskussion bereits abgelehnt wurde.

Grundsätzlich sollte im Genehmigungsantrag immer auf Wirksamkeitsnachweise mit möglichst hoher Evidenz verwiesen werden.

\section{Formalvorgaben bei der Antragstellung}

In vielen Fällen ist es wünschenswert, dass die Therapie mit einem Cannabinoid möglichst zeitnah begonnen werden kann. Dementsprechend sollten Verzögerungen durch Formalaspekte möglichst vermieden werden. Wichtig ist es daher, dass bei der Antragsstellung auch die folgenden Punkte beachtet werden:

\section{Versicherter ist zuständig für die Antrag- stellung}

Nach dem Wortlaut des Gesetzes ist allein der Versicherte zuständig für die Antragstellung nach §31 Abs.6 SGB V. Antragstellungen allein durch den behandelnden Arzt können daher zu Rückfragen führen, ob der Arzt bevollmächtigt ist, für den Patienten zu handeln, der eigentlicher Antragsteller ist. Soweit der Vertragsarzt daher im Namen des Patienten allein eine Antragstellung vornehmen möchte, sollte dem Antrag immer eine Vollmacht des Patienten vorgelegt werden. Ohne Vertretungsvollmacht ist der behandelnde Arzt nicht berechtigt, einen Genehmigungsantrag bei der Krankenkasse für einen Patienten zu stellen.

Der Arztfragebogen dagegen muss immer vom behandelnden Arzt ausgefüllt und dem Antrag beigefügt werden.

\section{Gesetzliche Genehmigungsfiktion des $\S 13$ Abs.3a SGB V}

Durch einen Verweis in $\S 31$ Abs.6 SGB V auf § 13 Abs.3a SGB V gilt auch für die Antragstellung zur Genehmigung einer Cannabinoid-Behandlung eine Genehmigungsfiktion bei nicht rechtzeitiger Rückmeldung der Krankenkasse.

Verpflichtet ist die Krankenkasse danach, über einen Antrag auf Leistungen zügig, spätestens bis zum Ablauf von drei Wochen nach Antragseingang oder in Fällen, in denen eine gutachtliche Stellungnahme, insbesondere des Medizinischen Dienstes der Krankenversicherung (Medizinischer Dienst), eingeholt werden muss, innerhalb von fünf Wochen nach Antragseingang zu entscheiden. Diese Frist ist - soweit eine Behandlung im Rahmen des SAPV, d. h. im Rahmen der Palliativversorgung erfolgt - auf 3 Tage verkürzt.

Wird dem Patienten innerhalb dieser Frist keine Entscheidung bekannt gegeben, so gilt der Antrag kraft Gesetzes als genehmigt.

In Bezug auf den Fristablauf ist jedoch die Betrachtung des Einzelfalls wichtig. Die Frist wird in den Fällen gehemmt, in denen die Krankenkasse ergänzende Unterlagen beim Versicherten angefordert hat. Die Frist läuft daher soweit und solange nicht weiter bis diese Unterlagen eingereicht sind. Diese zusätzlichen Zeiten müssen daher bei der Berechnung, wann die Genehmigungsfiktion eintritt, berücksichtigt werden. 
Eine nachträgliche Reaktion, d. h. eine Begrenzung oder Ablehnung von Anträgen ist dagegen - nach Ansicht der Rechtsprechung [12] - nicht mehr möglich, wenn die Genehmigungsfiktion einmal eingetreten ist. Die Richter stellten klar, dass nach den Vorgaben des Gesetzes eine durch die Fiktion eingetretene Genehmigung nur zurückgenommen werden könne, wenn neue, nach der Genehmigung eingetretene Tatsachen dies rechtfertigen. Anderenfalls habe die Genehmigungsfiktion Bestand.

\section{Möglichkeiten einer zeitlichen Befristung der Genehmigung}

In den vergangenen Monaten konnte immer wieder beobachtet werden, dass Krankenkassen versucht haben, Genehmigungen einer Therapie nach §31 Abs.6 SGB V zeitlich zu befristen. Die Rechtsprechung hat diesen Versuch im Ergebnis aber in einer Eilentscheidung [13] abgelehnt: Festgestellt wurde, dass eine Genehmigung der Krankenkasse nur bei einer erstmaligen Verordnung vorgesehen ist und dass dem Gesetz keine Rechtsgrundlage für eine Befristung zu entnehmen ist. Ärzten, die im Umgang mit von der Krankenkasse bereits mitgeteilter Befristungen unsicher sind, ist zu empfehlen, im Klagewege unmittelbar feststellen zu lassen, dass eine solche Befristung nicht rechtswirksam ist. Soweit die Widerspruchsfrist bereits verstrichen ist, sollte ein erneuter Antrag unter Hinweis auf die bisherige Wirksamkeit einerseits und die Rechtsprechung, die keine erneute Befristung zulässt, gestellt werden.

Abschließend ist festzustellen, dass in der nächsten Zeit noch diverse Streitfragen zu den Anträgen nach §31 Abs.6 SGB V durch die Gerichte entschieden werden müssen und dass kontinuierlich mehr Rechtssicherheit entstehen wird. Auf bereits entschiedene Streitfragen kann der Versicherte - bzw. auch der Arzt im Rahmen des Arztfragebogens durch Hinweis auf ein entsprechendes Gericht und Aktenzeichen verweisen, um erneute Diskussionen möglichst im Keim zu ersticken.

Unabhängig davon ist es ratsam, den vollständigen Therapieverlauf - einschließlich unerwünschter Wirkungen und Kontraindikationen - sehr detailliert im Genehmigungsantrag bzw. in der Patientenakte zu dokumentieren. Je umfangreicher das Vorliegen der Genehmigungsvoraussetzungen des §31 Abs.6 SGB V bereits bei der Antragstellung erfolgt, umso geringer ist die Wahrscheinlichkeit, dass Rückfragen gestellt werden oder der Rechtsweg beschritten werden muss.

\section{Interessenkonflikt}

Die Autorin gibt an, dass kein Interessenkonflikt besteht.
Autorin

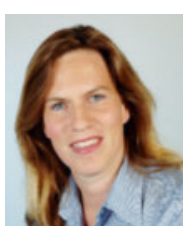

\section{Isabel Kuhlen}

Isabel Kuhlen wurde nach ihrer Approbation und jahrelanger Berufserfahrung als Apothekerin 2001 auch als Rechtsanwältin zugelassen. Mehrere Jahre war sie in der auf Medizinrecht und Pharmarecht spezialisierten Kanzlei von RA Herbert Wartensleben tätig. Von 2006 bis 2010 war sie in der Kanzlei Erckens, Horn und Partner in Mönchengladbach tätig. Seit August 2010 ist sie Mitbegründerin der Kanzlei Kuhlen.

Rechtsanwältin Kuhlen hält regelmäßig Vorträge zu medizinrechtlichen Fragestellungen und veröffentlicht Beiträge in medizinischen und juristischen Fachzeitschriften.

Korrespondenzadresse

Isabel Kuhlen
Rechtsanwältin und Apothekerin
Kanzlei Kuhlen
Rathausplatz 4
34246 Vellmar
E-Mail: isabel.kuhlen@kanzlei-kuhlen.de

Literatur

[1] LSG Hessen: Beschluss vom 04.10.2017; Az.: L 8 KR 255/17 $B E R$

[2] BSG Entscheidung vom 19.03.2002; Az.: B 1 KR 37/00 R

[3] BSG Entscheidung vom 27.03.2017; Az.: B 1 KR 30/06 R

[4] BSG Entscheidung vom 26.09.2006; Az.: B 1 KR 3/06 R

[5] SG Bremen: Beschluss vom 24.10.2017; Az.: S 7 KR 227/17 ER

[6] Begutachtungsanleitung Richtlinie des GKV-Spitzenverbandes nach §282 SGB V: „Sozialmedizinische Begutachtung von Cannabinoiden nach §31 Abs.6 SGB V“

[7] $\S 6$ (Muster)-Berufsordnung für die in Deutschland tätigen Ärztinnen und Ärzte - MBO-Ä - in der Fassung des Beschlusses des 118. Deutschen Ärztetages 2015 in Frankfurt am Main

[8] §4 Abs.13 AMG

[9] §33 AM-RL

[10] LSG Hessen: Beschluss vom 21.11.2017; L 8 KR 406/17 B ER

[11] BSG: Entscheidung vom 31.05.2006; Az.: B 6 KA 53/05 B

[12] SG Aachen: Az.: S 13 KR 476/17

[13] SG Hildesheim: Entscheidung vom 21.11.17 - Az.: S 32 KR 4041/17 ER

Bibliografie

DOI https://doi.org/10.1055/a-0606-9912

EHK 2018; 67: 128-132

(c) MVS Medizinverlage Stuttgart GmbH \& Co. KG ISSN 0014-0082 